\title{
New Technologies for Magnetic Resonance Imaging Compatible Device of Boston Scientific
}

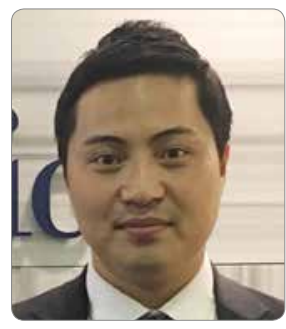

정지운

보스턴사이언티픽코리아

Ji Woon Jung

Boston Scientific Korea, Seoul, Republic of Korea

Received: February 19, 2016

Revision Received: April 18, 2016

Accepted: April 18, 2016

Correspondence: Ji Woon Jung, IBHRE, CCDS

Boston Scientific Korea 19F, SEl Tower, 39, Eonju-

ro 30-gil, Gangnam-gu, Seoul 06292, Republic of

Korea

Tel: +82-10-2333-8468 Fax: +82-2-3483-1822

E-mail: jjwcool@gmail.com

Copyright (C) 2016 The Official Journal of Korean Heart Rhythm Society Editorial Board \& MMK Co., Ltd.

\begin{abstract}
The use of both magnetic resonance imaging (MRI) and pacing devices has vastly increased worldwide in recent years. A significant number of implanted patients will likely need monitoring using magnetic resonance imaging (MRI) over the course of the lifetime of their device. Some studies have demonstrated that with appropriate precautions, MRI can be safely performed in patients with selected implantable pacemakers. However, MRI is still contraindicated in patients with pacemakers. Recently, new pacing systems have been specifically designed for safe use in the MRI environment. The first reported experience suggests that the technology is safe and may allow patients with these new pacemakers to undergo MRI. This review will describe the outstanding issues and controversies surrounding the safety of MRI in patients with pacemakers, and the potential benefits of the new MRI-conditional technology. We will also discuss how to decide whether an MRI-conditional system should be implanted, and highlight key issues that warrant further studies.
\end{abstract}

Key Words: - Magnetic Resonance Imaging • Pacemaker • Safety

\section{서론}

세계적으로 MRI (magnetic resonance imaging) 장비와 부정맥 치료용으로 사용되는 CIED (cardiovascular implantable electronic device) 제품의 사용은 지속적으로 증가하고 있다. 과거에는 $\mathrm{MRI}$ 촬영 시 발생할 수 있는 여러 가지 위험성 때문에 $\mathrm{CEED}$ 장치를 이식한 환자에서 촬영이 금지되어 있었으나, 최근에는 조건에 따라 촬영이 가능한 $\mathrm{CIED}$ 제품들이 출시되어 $\mathrm{CIED}$ 이식 환자라도 특정 조건 아래에서 MRI 촬영이 가능하게 되었다. ${ }^{1}$

\section{자기 공명의 원리와 이식형 기기에 미치는 영향}

$\mathrm{MRI}$ 촬영 장비는 크게 3 가지 주요 요소로 이루어져 있다 (Figure 1). ${ }^{2} \mathrm{MRI}$ 장비의 구성을 보면 RF (radiofrequency)coil, gradient coil, magnet으로 구성되어 있으며, MRI 촬영 시에 발생되는 전압이나 전류, $\mathrm{RF}$ 등은 $\mathrm{CEED}$ 제품 작동에 치명적인 오류나 이상을 일으킬 수 있다. MRI 촬영 시 $\mathrm{MRI}$ 장비를 구성하는 3 가지 주요 요소로부터 유발될 수 있는 $\mathrm{CIED}$ 에서의 위험은 아래와 같이 다양하다. 첫 번째로는 static magnetic field로 인해 유발되는 증상으로 $\mathrm{CIED}$ 의 
stimulation을 억제하거나 PG (pulse generator)의 프로그램을 reversion할 수 있다(Figure 2). ${ }^{2}$ 두 번째로는 MRI 촬영 시에 $\mathrm{RF}$ coil에서 $\mathrm{RF} \mathrm{B} 1$ 에 해당하는 에너지가 발생하는데, 이로 인해 인체에 삽입된 전극선이 가열되면서 그 기능에 영향을 주거나 전극선 주변의 조직들에 열이 가해져 loss of capture의 원인이 되기도 한다(Figure 3). 세 번째로 gradient coil에서 발생되는 에너지가 전극선과 $\mathrm{PG}$ 에 고전류를 흐르게 하여 회로의 이상이나 오동작을 유발하여 치명적인 부정맥을 발생시키기도 한다(Figure 4).
최근에 출시되는 보스톤사이언티픽의 $\mathrm{CED}$ 제품들은 오랜 기간의 연구를 통해 MRI 촬영 시 발생할 수 있는 여러 위험 요소에도 CIED 이식 환자가 안전할 수 있도록 전극선의 디자인 변화, $\mathrm{PG}$ 의 주요 부품 변경 및 삽입을 통해 발전해왔다. 현재 심박동기(permanent pacemaker)는 $3.0 \mathrm{~T}$, 삽입형 제세동기(implantable cardioverter-defibrillator, $\mathrm{ICD}$ )와 심장 재동기화 치료(cardiac resynchronization therapy, CRT X4 [4극 제품])는 각각 $1.5 \mathrm{~T}$ 의 MRI 장비에서 전신 촬영이 가능하고, 촬영 시간의 제한도 없는 제품들이

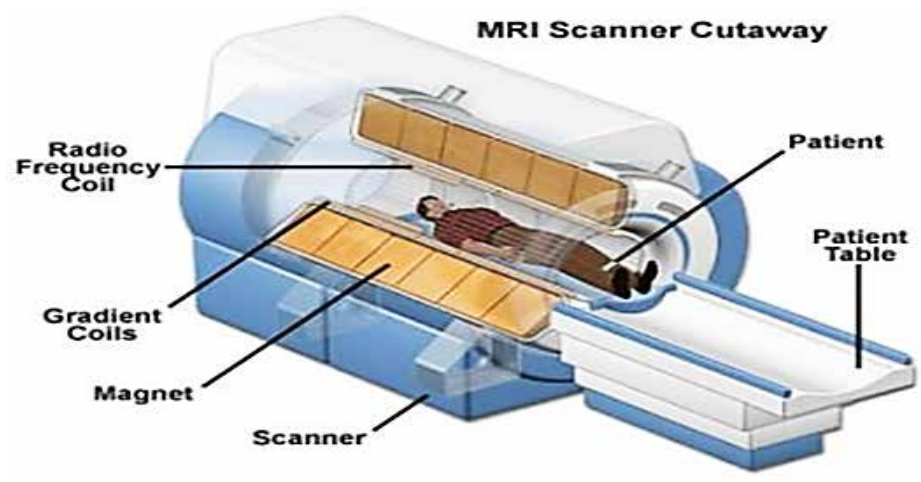

Figure 1. An magnetic resonance imaging scan generates 3 field: radiofrequency coil, gradient coils, magnet.

$\mathrm{MRI}$, magnetic resonance imaging.

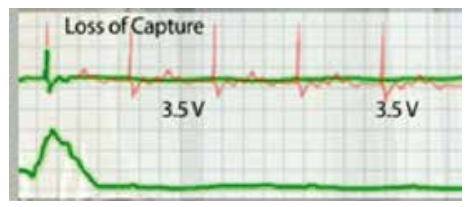

Figure 2. Static magnetic field (B0) effect on cardiovascular implantable electronic device.

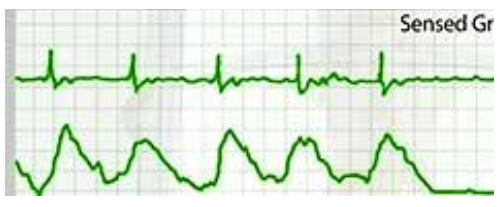

Figure 3. The radiofrequency magnetic field (B1) effect on cardiovascular implantable electronic device.

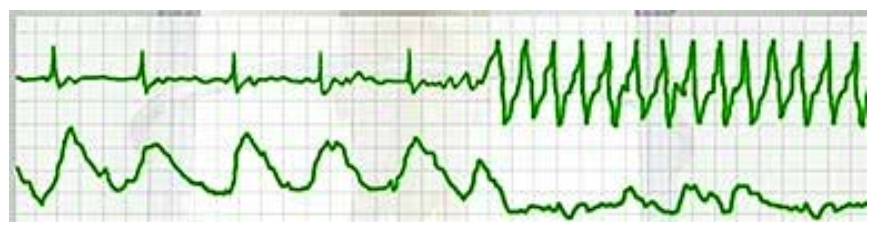

Figure 4. The magnetic gradient (Gx, Gy, Gz) effect on cardiovascular implantable electronic device. 
출시, 사용되고 있다.

\section{$\mathrm{MRI}$ 조건에 적합한 전극선 디자인}

앞서 $\mathrm{MRI}$ 촬영 시 생성되는 $\mathrm{RF}$ 로 인해 전극선에 전류가 흐르면서 전극선이 가열되고, 발생된 열로 인해서 주변 심장 조직에 영향을 미치며 역치 값이 높아지는 현상이 발생한다고 언급하였다. 이에 보스톤사이언티픽은 전극선에 강한 전류가 흐르더라도 열의 발생을 줄이는 새로운 전극선 디자인을 고안하였다(Figure 5). ${ }^{3}$ 전극선의 디자인에 따라 MRI 촬영 시 $\mathrm{RF}$ 에 의해 열이 더 많이 발생하거나 더 적게 발생할 수 있다. 전극선의 내부는 일반적으로 코일이 감겨 있는 형태로 이루어져 있는데, 코일의 지름이 크고, 간격이 좁고, wire의 지름이 작을수록 $\mathrm{RF}$ 에 의한 열은 감소한다. 반면 코일의 지름이 작고, 간격이 넓으며, wire의 지름이 크면 $\mathrm{RF}$ 에 의해 발생하는 전극선의 열은 더 크게 발생한다. 현재 보스톤사이언티픽에서 출시된 INGEVITY ${ }^{\mathrm{IM}}$ 전극선은 코일의 지름이 크고, 코일의 간격은 매우 촘촘하게 좁으며, wire
지름은 작고 single filar coil 형태로 디자인되어 MRI 촬영 시 생성되는 RF가 전극선에 흐르더라도 발열을 최소화하도록 설계되었다(Figure 6). Dual filar 형태나 multi-filar coil의 형태로 제작된 전극선은 코일 간격이 더 넓어 MRI 촬영 시에 $\mathrm{RF}$ 가 생성되면 넓은 코일 간격으로 인해 전극선 내부와 끝 부분에 온도가 높아지기 때문에 single filar coil 형태를 채택하였다.

\section{$\mathrm{MRI}$ 조건에 적합한 내재성 Pacemaker 디자인 요소}

$\mathrm{MRI}$ 촬영 시 생성되는 $\mathrm{RF}$ 로 인해 열이 발생하는 부작용은 새로운 전극선 디자인의 설계로 해결하였으나 여전히 gradient coil에서 발생하는 고전류로 인해 pacemaker에 문제가 발생할 수 있다. 이를 보안하기 위해 보스톤사이언티픽의 permanent pacemaker 장치에는 surge suppressor라는 부품이 추가 삽입되어 MRI 촬영 시 고전류가 pacemaker에 전달되더라도 안정적으로 전류를 공급하도록 유지한다(Figure 7).

\section{Low Inductance Coil}
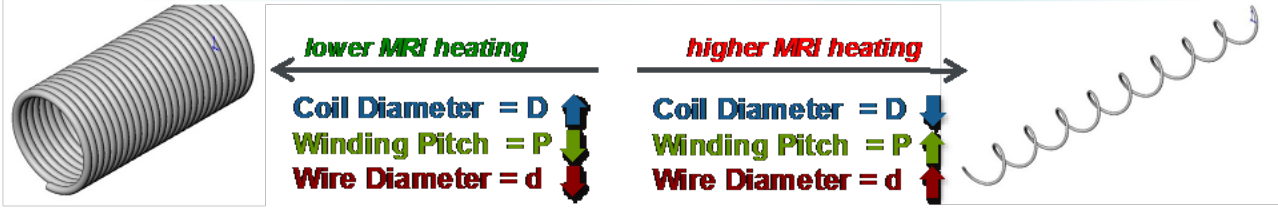

\section{Coill designs across industry}

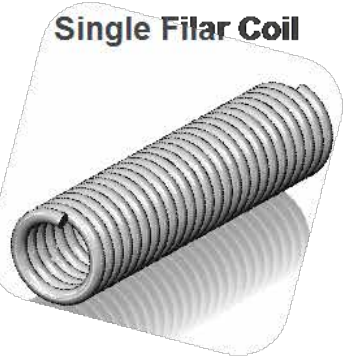

Dual Filar Coil

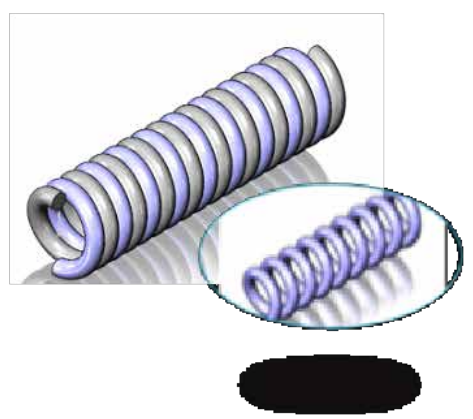

Multi-Filar Coil

(4 filar)

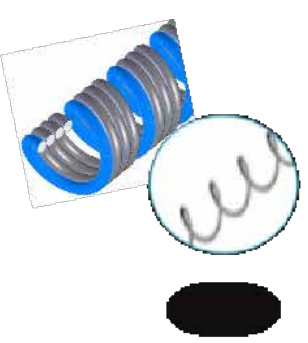

Figure 5. Magnetic resonance imaging Lead Design for lower heating. 


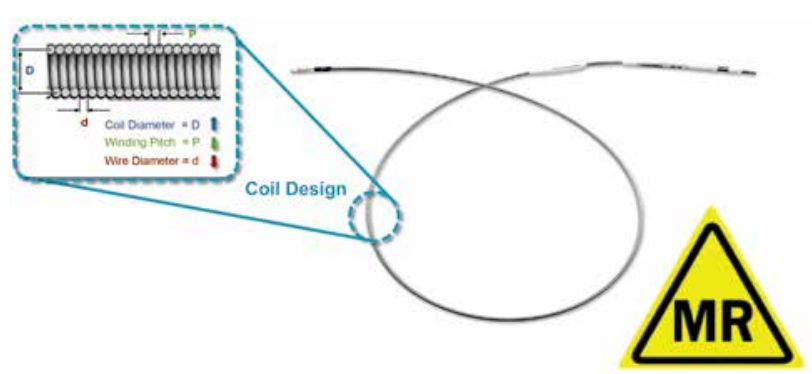

Figure 6. Design of IngevityTM lead for $3 \mathrm{~T}$ magnetic resonance imaging condition.

MRI 촬영 시 gradient coil에서 발생하는 높은 전압이 pacemaker에 전달되면 surge 현상으로 인해 pacemaker가 비정상적으로 동작하거나 회로의 고장을 유발한다. Surge는 갑작스럽게 높은 전압이 유입되는 현상을 의미하며, 일반적으로 surge 증상이 기계 장치에 발생하면 장치의 회로가 고장 난다. 보스톤사이언티픽의 permanent pacemaker는 갑작스럽게 높은 전압이 내부 회로에 흘러도 surge suppressor를 통해 안정적으로 전압을 유지한다. Surge suppressor와 같이 pacemaker 내부의 다른 주요 부품들도 MRI 촬영이 가능하도록 구성을 변경하였는데, 촬영 시 미치는 영향을 최소화하도록 배터리 재질도 기존에 사용되던 니켈(nickel)에서 스테인리스 스틸(stainless steel)로 변경하였다. B0 (static magnet field)으로 인해 생성되는
자력으로 인해 발생하는 부작용을 최소화하였고, MICS (medical implant communication service) 안테나를 삽입하여 프로그래머와 무선 통신이 가능하다. 또한 기기 구성의 변경을 통해 RF가 기계 내부에 흐를 때 미치는 영향을 줄이며, EMI (electromagnetic interference) 필터가 pacemaker 안에 장착되어 $\mathrm{RF}$ 로 인해 발생하는 $\mathrm{EMI}$ 를 감소시키고, $\mathrm{PG}$ 헤더 부분에 위치한 전극선 고정부위의 재질을 변경하고, 아날로그 IC (integrated circuit) 전자 회로를 사용하여 gradient coil로 인해 발생되는 전류의 흐름에 의한 영향을 최소화하였다. ${ }^{4}$

Permanent pacemaker에는 surge suppressor가 삽입되어 있으나, ICD와 CRT 제품에는 surge suppressor가 삽입되지 않았다. ${ }^{5}$ 그러나 나머지 부품들은 high voltage의 특성에 맞추어 MRI의 영향을 최소화하는 부품으로 변경하였다 (Table 1). 또한 조건적으로 MRI 촬영이 가능한 pacemaker는 $\mathrm{X}$-ray $\mathrm{ID}$ 만으로 알 수 있도록 $\mathrm{PG}$ 내에 위치한 $\mathrm{D}$ 부분에 tag 를 달았다. 응급상황이나 환자가 이식한 기계에 대한 정보가 없더라도 단순 $\mathrm{X}$-ray 촬영으로 $\mathrm{MRI}$ 촬영이 조건에 따라 가능한 pacemaker인지를 판별이 가능하다. 보스톤 사이언티픽의 모든 pacemaker는 X-ray tag가 삽입되어 있는데, $\mathrm{MRI}$ 촬영이 가능한 제품의 경우 가장 앞쪽에 삼각형 모양이 삽입되어 있다(Figure 9).

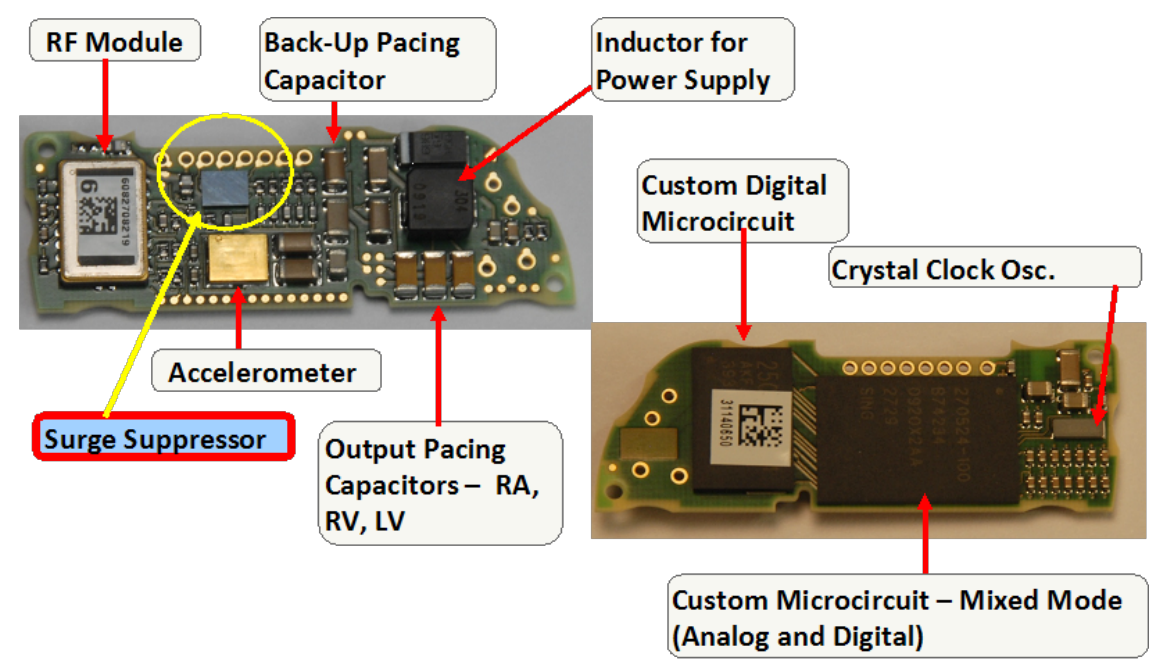

Figure 7. Surge suppressor in pacemaker.

$\mathrm{LV}$, left ventricle; $\mathrm{RA}$, right atrium; $\mathrm{RF}$, radiofrequency; $\mathrm{RV}$, right ventricle. 


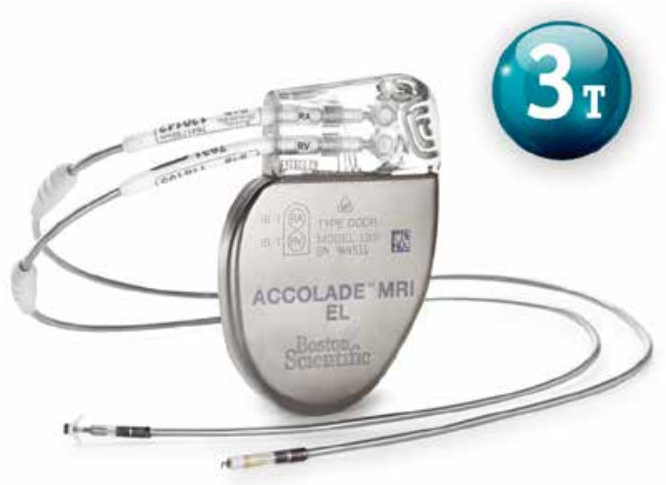

Figure 8. Magnetic resonance imaging pacemaker system.

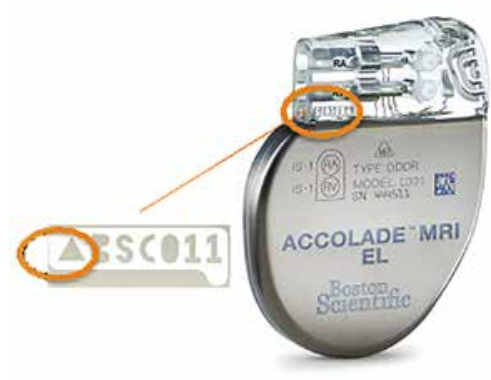

Figure 9. A specific triangle that is added on the identifier that can be found in the header of the can.

Table 1. Inherent cardiovascular implantable electronic device design for magnetic resonance imaging environment (ICD, CRT)

\begin{tabular}{l|l}
\hline \multicolumn{1}{c|}{ Side A } & \multicolumn{1}{c}{ Side B } \\
\hline Digital integrated circuits ball grid array assembly & Analog integrated circuit Wafer Level Chip Scale Package (WLCSP) \\
Radiofrequency module & Super output module \\
$\begin{array}{l}\text { Accelerometer } \\
\begin{array}{l}\text { Discrete electronic components (resistors, diodes, capacitors, crystal oscilla- } \\
\text { tor, inductors) }\end{array}\end{array}$ & High voltage charge module \\
\hline
\end{tabular}

CRT, cardiac reorganizations therapy; ICD, implantable cardioverter-defibrillator.

\section{$\mathrm{MRI}$ 스캔에 적합한 CIED 시스템 관리}

$\mathrm{MRI}$ conditional 제품의 경우 MRI를 촬영하기 위해 관련된 필요한 조건의 확인이 반드시 필요하다.

- $\mathrm{CIED}$ 시술 후 최소 6 주 이상 경과한 환자

- Pectoral implantation

- No abandoned leads, lead extenders or adapters

- Lead electrically intact, with stable and normal function

- Lead impedance between 200 and 1,500 ohm

- Capture threshold <2.0 V/0.4 msec

$\mathrm{MRI}$ 사용이 가능한 $\mathrm{CIED}$ 제품이라도 상기 조건에 해당하는 환자만 $\mathrm{MRI}$ 촬영이 가능하며, 각 $\mathrm{CIED}$ 제품에 따라 촬영 부위가 전신 또는 신체의 일부분만 촬영이 가능한지, 촬영 시간의 제한이 있는지, MRI의 종류에 있어 $1.5 \mathrm{~T}$ 또는
$3.0 \mathrm{~T}$ 에서 촬영이 가능한 $\mathrm{CEED}$ 제품인지 확인이 필요하다. 그리고 촬영 전에 $\mathrm{MRI}$ mode로 변경하여 촬영하여야 한다. $\mathrm{CIED}$ 제품을 MRI mode로 변경하는 방법은 출시된 회사마다 차이가 있다. 보스톤사이언티픽의 경우에는 'MRI protection time-out' 기능이 내장되어 있어서 MRI mode로 변경한 이후 설정된 시간이 지나게 되면 변경 전 설정으로 자동 변경된다 (Figure 10). ${ }^{8}$

\section{결론}

$\mathrm{MRI}$ 촬영이 보편화되었지만, 불과 몇 년 전까지만 하더라도 $\mathrm{CIED}$ 이식 환자들은 $\mathrm{MRI}$ 촬영에 대한 안전을 보장받지 못하여 MRI 촬영이 불가능했다. 하지만 지금은 관련 회사들의 여러 노력으로 인하여 조건부로 $\mathrm{MRI}$ 촬영이 가능한 제품들이 등장하였고, 앞으로는 이보다 더 발전된 기술의 제품이 출시되기를 기대해 본다. 


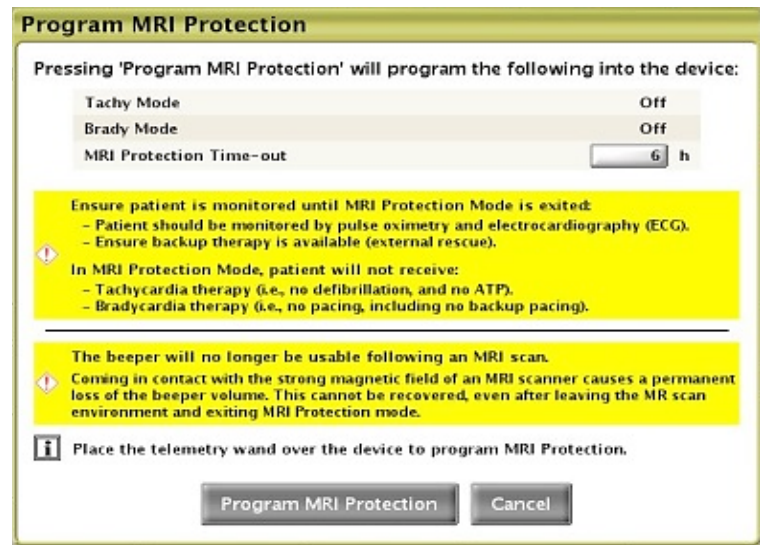

Figure 10. Magnetic resonance imaging (MRI) protection mode.

\section{References}

1) Santini L, Forleo GB, Santini M, World Society of Arrhythmias. Evaluating MRI-compatible pacemakers: patient data now paves the way to widespread clinical application. Pacing Clin Electrophysiol. 2013;36:270-278.

2) Boston Scientific. ANZ_PSST_13009_ImageReady technical training_25Feb2013 [Data on file].

3) Boston Scientific. INGEVITY PTM 359069-001 Fineline-ingevity MRI technical guide [Data on file].

4) Boston Scientific. INGENIO pacemaker system MRI design summary (100051-698) Rev B [Data on file].

5) Boston Scientific. CRM-263902-AA_AUG2014_ACCOLADE_ (only)_inservice_final_TTT[Data on file].

6) Boston Scientific. AEMA MRI Ask the experts ANZ_ PSSP_13037 EMEA MRI ask the experts [Data on file].

7) Park JW. MRI compatible pacemaker: current perspectives [in Korean]. Korean Heart Rhythm Society Symposium; 2015; Ilsan, Goyang, Republic of Korea.

8) Boston Scientific. CRM-329418-AA JUL 2015 Tachy MRI in-service green [Data on file]. 